\title{
Thermal electronic properties of alkali clusters ${ }^{\star}$
}

\author{
M. Brack ${ }^{1}$, O. Genzken ${ }^{1}$, and Klavs Hansen ${ }^{2}$ \\ ${ }^{1}$ Institut für Theoretische Physik, Universität Regensburg, W-8400 Regensburg, Federal Republic of Germany \\ 2 The Niels Bohr Institute, Blegdamsvej 17, DK-2100 Copenhagen, Denmark \\ Received 10 September 1990
}

\begin{abstract}
We apply the finite-temperature Kohn-Sham method to alkali metal clusters, using the spherical jellium model and treating the valence electrons as a canonical system in the heat bath of the ions. We study the shell effects in the total free energy $F(N)$ and the entropy $S(N)$ for neutral clusters containing $N$ atoms. Their strongest temperature dependence is due to the finite ground-state value $S_{0}>0$ of the electronic entropy for non-magic clusters. It leads to a decreasing amplitude and an increasing smear-out of the saw-tooth structure in the first difference $\Delta_{1} F(N)=F(N-1)-F(N)$ with increasing temperature $T$ and cluster size $N$.
\end{abstract}

PACS: $36.40 .+\mathrm{d} ; 31.20 . \mathrm{Sy} ; 05.30 .-\mathrm{d} ; 65.60 .+\mathrm{m}$

Since the discovery of shell effects in metal clusters, much effort has gone into their theoretical understanding. Until now, all calculations of electronic shell structure have been restricted to $T=0 \mathrm{~K}$. In order to compare with experiments, however, it is necessary to include finite temperature effects. These turn out to be surprisingly large due to the dual effect of shell structure in providing both an energy gap at the major shell closings and a high electronic degeneracy of clusters with non-closed shells.

In a recent paper [1] we have applied selfconsistent microscopical calculations for metal clusters at $T>0$, using the finite-temperature Kohn-Sham (KS) density functional formalism [2]. We use the spherical jellium model and the local-density approximation as in [3] to write the total free energy of a neutral cluster with $N$ atoms in terms of the local electron density $\rho$ as $F[\rho]$ $=U[\rho]-T S[\rho]$. Here $U[\rho]$ is the internal energy (including that of the ions in the jellium approximation) which for $T=0$ is identical to the ground-state energy

\footnotetext{
* Work partially supported by the Danish Natural Science
} Research Council and by Deutsche Forschungsgemeinschaft
$E[\rho]$ obtained in earlier KS calculations [3]. The new ingredients here are the entropy $S$ of the electrons and the finite-temperature occupation numbers $n_{i}$ entering the electron density $\rho(r)=\sum\left|\varphi_{i}(\mathbf{r})\right|^{2} n_{i}$ with $\int \rho(r) \mathrm{d}^{3} r=\sum_{i} n_{i}$ $=N$. The single-particle wavefunctions of the electrons are obtained by solving iteratively the KS equations $\left\{\hat{T}+V_{\text {tot }}[\rho(r)]\right\} \varphi_{i}(\mathbf{r})=\varepsilon_{i} \varphi_{i}(\mathbf{r})$. At each iteration, the occupation numbers $n_{i}$ are evaluated from the canonical partition function $Z_{N}(\beta)$ (with $\beta=1 / T$ ) which we calculate exactly in terms of the s.-p. levels $\varepsilon_{i}$ (see [1] for details). After convergence, we also obtain the entropy $S$ as well as the non-interacting parts of the energies $F$ and $U$ from $Z_{N}$ by the usual quantum statistical relations.

As a result of our numerical calculations we find that the total mean field $V_{\text {tot }}(r)$ and with it the density $\rho(r)$ and the s.-p. levels $\varepsilon_{i}$ depend very little on temperature up to several thousand Kelvin. The temperature dependence of the free energy is governed by the term - TS, and thus by the entropy of the electrons, as long as $T$ is much smaller than the major shell gaps.

In Fig. 1 we show the oscillating part, $\delta F(N)=F(N)$ $-\tilde{F}(N)$, of the total free energy versus $N^{1 / 3}$ at two temperatures. $\delta F$ is defined, in the spirit of Strutinsky's nuclear shell-correction theory [4], as the difference between the exact microscopic total free energy $F(N)$ and a smooth part $\widetilde{F}(N)$. Note the sharp minima of $\delta F$ at the magic numbers which correspond to filled major electronic shells at $T=0$. The amplitude of the oscillations is clearly reduced with increasing temperature and cluster size.

We show in Fig. 2 the entropy per electron $S / N$, plotted versus $N^{1 / 3}$. It exhibits dramatic shell effects. For systems with magic numbers, the ground state is nondegenerate and the $T=0$ entropy $S_{0}$ is zero. For open shells, $S_{0}=\log \left(\begin{array}{l}g \\ v\end{array}\right)>0$, where $g$ is the degeneracy of the uppermost level containing $v$ particles. Thus, the shell oscillatons in $S$ reflect the successive fillings of the degenerate spherical shells. At $T=400 \mathrm{~K}$, we find that the smaller clusters $(N \lesssim 60)$ still have approximately their ground-state entropy $S_{0}$. For larger $N$ (or higher $T$ ), the oscillations are damped and the entropy tends towards its 

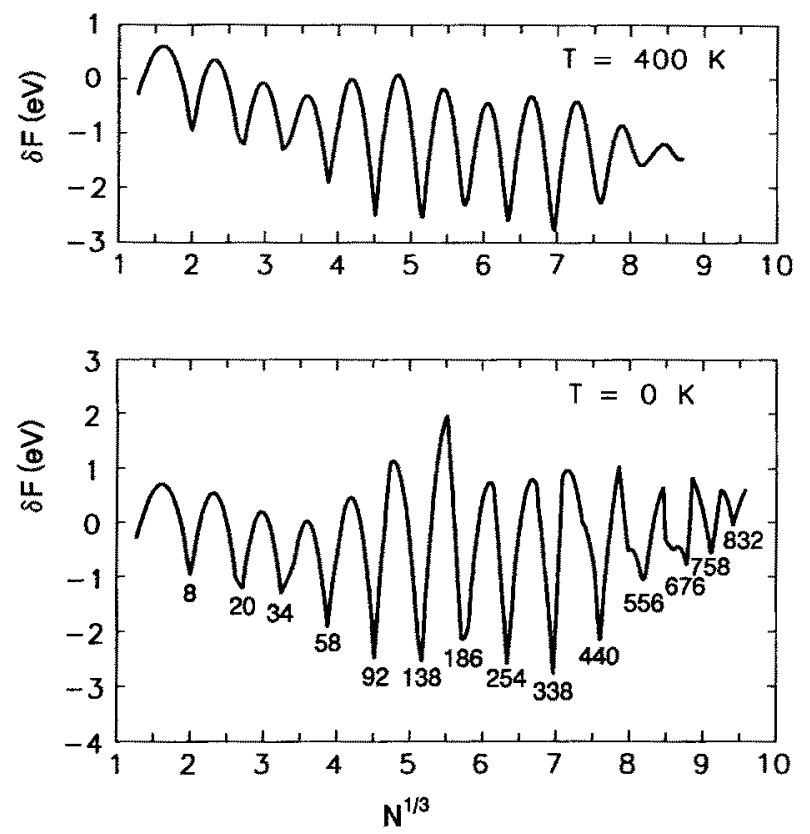

Fig. 1. Shell-correction $\delta F$ to the free energy versus $N^{1 / 3}$. Magic numbers for closed main shells are indicated at the bottom

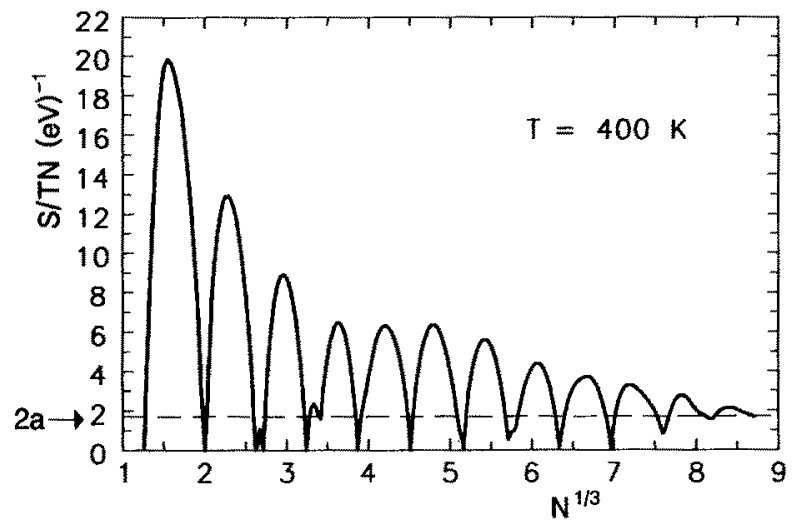

Fig. 2. Entropy per electron, $S / N$, versus $N^{1 / 3}$. (See text for the level density parameter $a$ )

linear behaviour $S \sim 2 a N T$ expected from the Fermi gas model.

In Fig. 3 we present the first differences $\Delta_{1} F(N)=$ $F(N-1)-F(N)$ and the second differences $\Delta_{2} F(N)=$ $F(N+1)+F(N-1)-2 F(N)$ of the free energy versus atom number $N$. At $T=0, \Delta_{2} E$ has the familiar spikes at the magic numbers; at $T=400 \mathrm{~K}$ these are drastically reduced with increasing $N$. In chemical equilibrium, the electronic part of $\Delta_{2} F$ is related [5] to the second difference of the logarithm of cluster concentrations $c(\mathrm{~N})$ : $\Delta_{2} F(N) \simeq-T \Delta_{2} \log c(N)$ (assuming the ionic contribution to be a smooth function of $N$ ). The broadening and the decrease in amplitude of the spikes at closed shells signal the gradual disappearance of electronic shell structure. As can be seen in Fig. 3, the decrease is much faster than the $N^{-1 / 3}$ dependence expected [6] at $T=0 \mathrm{~K}$.
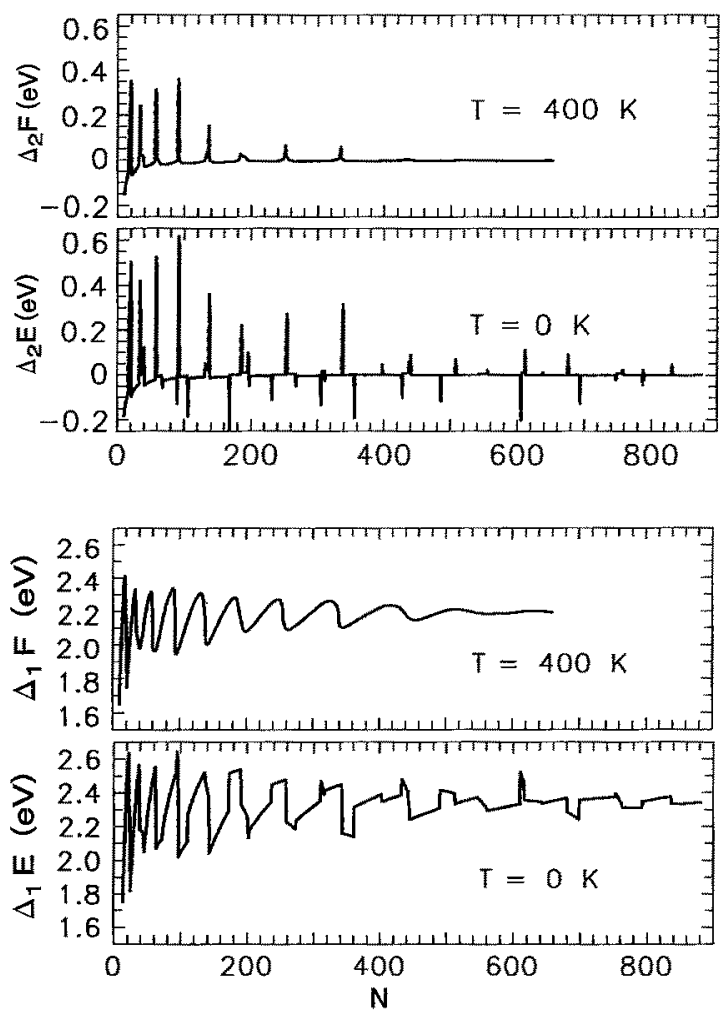

Fig. 3. First differences $\Delta_{1} F$ (lower part) and second differences $\Delta_{2} F$ (upper part) of free energy, plotted versus cluster size $N$. (The negative spikes in $\Delta_{2} E$ at $T=0$ are due to the imposed spherical symmetry and occur where the clusters would be deformed; see [1] for details.)

Typically, the shell effects vanish almost completely at a temperature of about one third of a major shell gap [1].

The electronic degrees of freedom in an isolated cluster can be treated in terms of a canonical ensemble. This is warranted by the very small amount of excitation energy carried by the electrons in comparison with the ions, which therefore act as a heat bath for the electrons. Even though the finite temperature effects have been calculated here for internal thermal equilibrium of isolated clusters, they are pertinent also for evaporation processes which appear to play an important role in determining the observed shell strucute in cluster abundance spectra from e.g. expansion sources [7]. If the transition state is assumed to be identical to the final state after evaporation, the $N$ dependence of the decay rate for an isolated cluster is determined essentially by the factor $\exp \left(-\beta \Delta_{1} F\right)$. The first difference $\Delta_{1} F(N)$ in Fig. 3 exhibits the familiar sawtooth structure at $T=0$. At $T=400 \mathrm{~K}$, this sharp structure is preserved up to $N \simeq 60$. With increasing $N$, however, it is gradually reduced in amplitude and the sawteeth are smeared out. This, again, is the effect of the degenerate (or nearly degenerate) shells on either side of the shell gaps in the spectrum $\varepsilon_{i}$.

The qualitative behaviour of the calculated $\Delta_{1} F(N)$ at $T=400 \mathrm{~K}$ bears a remarkable similarity to that of recently observed cluster abundance spectra [7]. Although the quantitative relation between $\Delta_{1} F\left(\right.$ and $\Delta_{2} F$ ) and these 
spectra is not yet fully understood, we infer from this similarity that the finite entropy of the valence electrons is essential in describing the thermal properties of metal clusters and appears, indeed, to be directly observable in such mass yields as found in [7].

Although the shell effects are to some extent exaggerated in our present model due to the imposed spherical symmetry, we expect that inclusion of deformational degrees of freedom will not alter seriously the qualitative picture obtained here, at least for the larger clusters with $N \gtrsim 100$

In [1], we discuss the Koopmans theorem at $T>0$ and related approximations allowing a fast numerical determination of $\Delta_{1} F$ and $\Delta_{2} F$; we also investigate in detail the differences between the canonical and the grand canonical description and their respective results, and present the asymptotic behaviour of entropy and specific heat of finite clusters.
We thank S. Bjørnholm for his continuous support and interest, and J. Borggreen for valuable criticism.

\section{References}

1. Brack, M., Genzken, O., Hansen, K.: Phys. Rev. B; (submitted for publication) Prepr. NBI-90-46

2. Gupta, U., Rajagopal, A.K.: Phys. Rep. 87, 259 (1982)

3. Ekardt, W.: Phys. Rev. B 29, 1558 (1984)

4. Strutinsky, V.M.: Nucl. Phys. A122, 1 (1968); see also Brack, M., Quentin, P.: Nucl. Phys. A361, 35 (1981)

5. Engelking, P.C.: J. Chem, Phys. 87, 936 (1987)

6. Nishioka, H., Hansen, K., Mottelson, B.R.: Phys. Rev. B, (in press); Z. Phys. D - Atoms, Molecules and Clusters (1991) (this issue)

7. Bjornholm, S., Borggreen, J., Echt, O, Hansen, K., Pedersen, J., Rasmussen, H.D.: Phys. Rev. Lett. 65, 1627 (1990); Z. Phys. D - Atoms, Molecules and Clusters (1991) (this issue) 